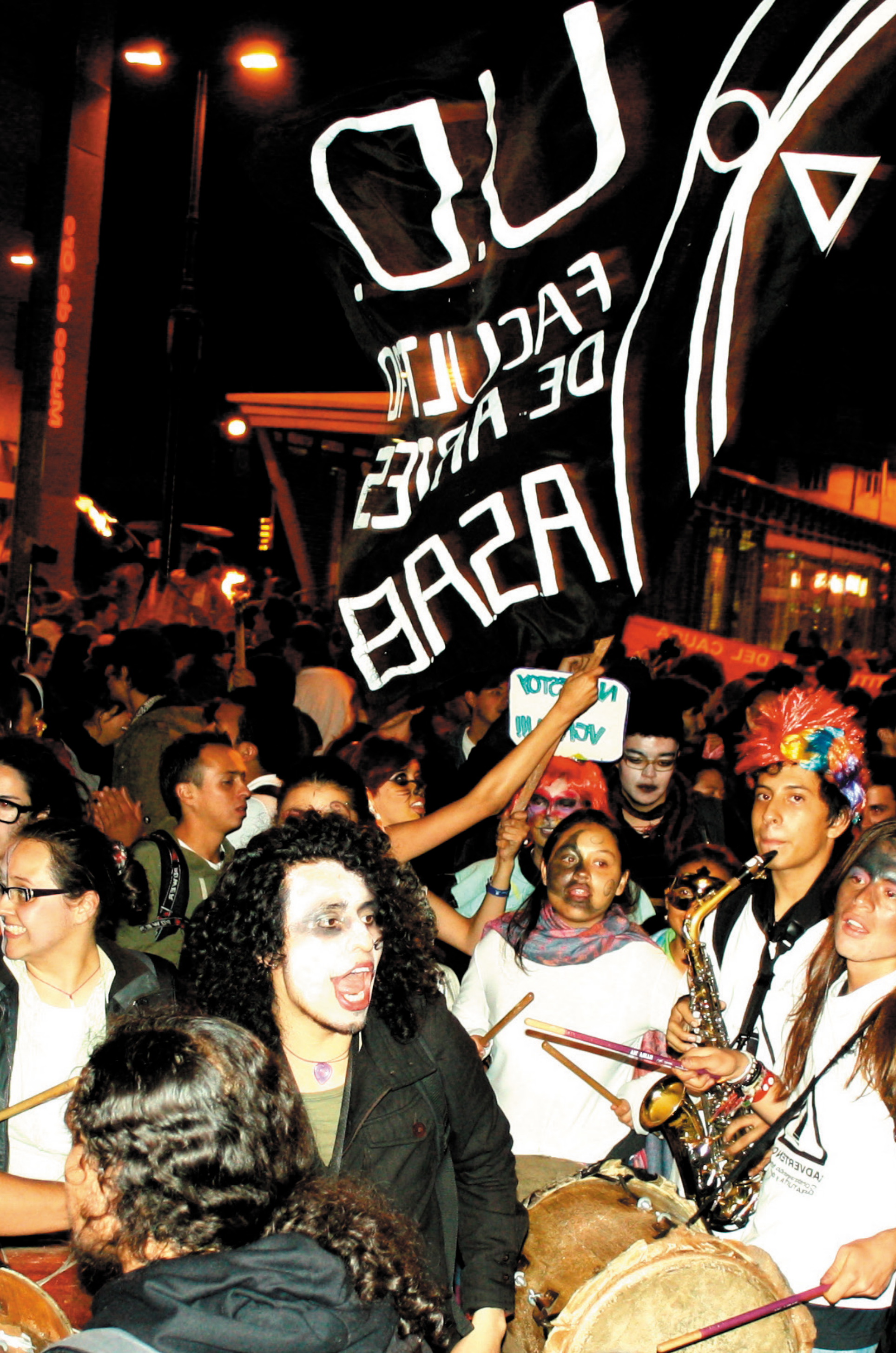




\title{
Arte y construcción de paz: la experiencia musical vital
}

\section{Artículo de reflexión}

\section{Gloria Patricia Zapata}

Fundación Universitaria Juan N. Corpas

glopaza@gmail.com

\begin{abstract}
(1) (1)
Cómo citar este artículo: Zapata, G. [2017]. Arte y construcción de paz: la experiencia musical vital, Calle14 revista de investigación en el campo del arte, 12(22), 240-253

DOl: https://doi.org/10.14483/21450706.12356
\end{abstract}

\section{Resumen}

Este artículo propone diversos elementos que contribuyen, desde las prácticas artísticas, a la construcción de paz. Partiendo de múltiples investigaciones, analiza el rol de las artes y, más específicamente, el papel de la experiencia musical vital, señalando niveles de investigación, ámbitos, teorías [como las ciencias cognitivas y la psicología del desarrollo], así como retos desde los cuales estas prácticas pueden cumplir un papel fundamental en dicha tarea. De esta forma, se clarifican los distintos aspectos en los que es preciso poner la mirada; con la fenomenología como soporte teórico del concepto de experiencia musical vital, se señalan algunos ámbitos posibles para la investigación, como también los dispositivos propios de las prácticas artísticas que son útiles en la construcción de paz. Con esto se espera aportar en el desarrollo del conocimiento sobre la relación entre arte y paz.

\section{Palabras claves}

Experiencia musical vital, prácticas artísticas, construcción de paz, experiencia humana. 


\section{Art and Peacebuilding: The Vital Musical Experience}

\section{Abstract}

This article proposes diverse elements that contribute, from the artistic practice, to the construction of peace. Based on multiple researches, it analyzes the role of the arts and, more specifically, the role of vital musical experience, pointing to levels of research, fields, theories [such as the cognitive sciences and developmental psychology] as well as challenges from which These practices can play a key role in this task. In this way, they clarify the different aspects where it is necessary to look. With phenomenology as a theoretical support of the concept of vital musical experience, some possible fields for research are pointed out, as well as the devices of the artistic practices that are useful in the construction of peace. This is expected to contribute to the development of knowledge about the relationship between art and peace.

\section{Keywords}

Vital music experience, artistic practice, peacebuilding, human experience.

\section{Art et construction de la paix : l'expérience musique vitale}

\section{Résumé}

Cet article propose divers éléments qui contribuent, dès les pratiques artistiques, à la construction de la paix. Sur la base de recherches multiples, il analyse le rôle des arts et, plus particulièrement, le rôle de l'expérience musicale vitale, indiquant des niveaux de recherche, des domaines, des théories [comme les sciences cognitives et la psychologie du développement] ainsi que les défis à partir desquels ces pratiques peuvent jouer un rôle clé dans cette tâche. De cette façon, il clarifie les différents aspects à regarder de près. Avec la phénoménologie en tant que support théorique du concept d'expérience musicale vitale, certains domaines possibles de recherche sont soulignés, ainsi que les dispositifs des pratiques artistiques utiles à la construction de la paix. Cela devrait contribuer au développement des connaissances sur la relation entre l'art et la paix.

\section{Mots clés}

Expérience musicale vitale, pratiques artistiques, consolidation de la paix, expérience humaine.

\section{Arte e construção de paz: a experiência musical vital}

\section{Resumo}

Este artigo propõe diversos elementos que contribuiem desde as praticas, à construção de paz. Partindo de Múltiplas investigações, analisa o papel das artes e, más especificamente, a da experiência musical vital, assinalando níveis de investigação, âmbitos, teorias [como as ciências cognitivas e a psicologia do desenvolvimento], assim como propósitos desde os quais estas praticas podem cumprir um papel fundamental em dita tarefa. Desta forma, se clarificam os diferentes aspectos nos que é preciso pôr a mira, com a fenomenologia como suporte teórico do conceito de experiência musical vital, se assinalam alguns âmbitos possíveis para a investigação, como também os dispositivos próprios das práticas artísticas que são úteis na construção de paz. Com isto se espera aportar no desenvolvimento do conhecimento sobre a relação entre arte e paz. 


\title{
Palavras-chave
}

Experiência musical vital, práticas artísticas, construção de paz, experiência humana.

\begin{abstract}
Arte y uinahil pazmanda: sug experiencia Tunai Vital

\section{Maillallachiska:}

Kai articulok proponekume diversos elementokuna que contribuyen, sug practica artistikunana, uiñachingapa paz kallarispa Achka investigacionkuna, analiza chi rol artekunape y más específicamente, sug papel experiencia tunaikuna vital, señalaska nivelkuna investigación, ámbitos, teorías [chi cienciakuna cognitiva y psicología del desarrollo], chasa retocena chimanda chi practicakuna cumplenkuna sug papel fundamental chi tareape. Chasapek clarifikarenme sugrigcha aspectokuna y ka preciso churangapa kauai, chi fenomenologiak sug soporte teorikocena de experiencia tunaikuna vital, kauachimi sug ambitokuna allikai investigacionpepa, chasallata chi dispositivo propio chi practika artistikakuna y ka ministido uiñachingapa paz. Kaiua suiarikumi aportangapa sug desarrollo resengapa chi realcion entre arte y paz.
\end{abstract}

\section{Rimangapa Ministidukuna:}

\author{
Experiencia tunaikuna vital,practicas artísticas, uiñachii paz, experiencia humana.
}

La construcción de paz es una labor compleja que implica un ejercicio maduro de ciudadanía y asumir retos que comprometan a todos los miembros de la sociedad. Por eso, al igual que otras iniciativas, es necesario identificar las diferentes perspectivas y entradas para alcanzar este objetivo en Colombia. Sin embargo, la construcción de paz depende de las situaciones que originaron el conflicto; por lo tanto, las acciones encaminadas a tal empresa deben desarrollarse en los diversos contextos en los cuales se evidencia la violencia, sus causas y las posibilidades de intervención, ya que cada escenario presenta desafíos diferentes.

En consecuencia, con base en un trabajo de reflexión sobre las prácticas artísticas, es pertinente considerar los diversos lenguajes y materiales que se utilizan [el sonido, el cuerpo, la palabra, la imagen, el movimiento, entre otros], para analizarlos en sus particularidades y estudiar lo específico de la experiencia musical y sus posibles efectos en la construcción de paz.

\section{Enfoques teóricos sobre los niveles de la experiencia humana}

Las ciencias cognitivas y la psicología del desarrollo proponen diversos niveles de análisis de la experiencia humana, los cuales interactúan de distintas maneras y de forma simultánea en la construcción de esa experiencia. Es muy difícil descomponerlos; debido a la complejidad de su estudio, se abordan por separado para lograr una mejor comprensión de los fenómenos subyacentes y las formas como influyen en la construcción de sentido y de realidad articulada de los seres humanos.

El nivel filogenético lo constituye la herencia biológica que poseemos como especie y se observa en los reflejos primarios o arcaicos de los recién nacidos, que permiten la construcción de esquemas y, de acuerdo con las interacciones del individuo, se despliegan como patrones de desarrollo. Desde este enfoque se explica al individuo como 
un sistema orgánico con una característica fundamental que es la auto-organización o autopoiesis (Maturana, 1976; 1997; 2002], por medio de la cual el sujeto se "acopla" al entorno a través de una red de procesos y relaciones. Esta biología de la cognición hace posible el desarrollo del pensamiento con base en el movimiento, lo que desde la perspectiva de Llinás serían los Patrones Fijos de Acción [PAF] y los cualias, como estrategia y función unificadora que permite la construcción de sujeto (Llinas, 2003). Es clave indicar que el ámbito filogenético brinda posibilidades y herramientas muy interesantes y poco exploradas en investigaciones acerca de la relación entre prácticas artísticas y construcción de paz.

Desde otro enfoque, pero también con una base biológica, Piaget asocia la experiencia humana con el aprendizaje, que es un proceso de asimilación-acomodación fundamental de acciones que generan esquemas. Según Piaget, estas acciones se dan en un proceso circular entre acción y experiencia, en el cual las acciones inicialmente son motrices y luego son mentales. Por ello, desde la perspectiva constructivista, "todo conocer es hacer y todo hacer es conocer" [Maturana, 1997). El nivel filogenético da cuenta, en parte, de las interacciones iniciales del sujeto con el ambiente, como en el caso de los estudios sobre el desarrollo de la musicalidad en bebés (Malloch y Trevarthen, 2009]. Sin embargo [como se dijo antes], el nivel filogenético de la experiencia musical ha sido muy poco estudiado en la relación entre arte y construcción de paz.

El nivel ontogenético comprende la construcción de la experiencia del individuo y se refiere a las etapas que este recorre durante su vida, como la infancia, adultez y vejez. Desde la perspectiva del constructivismo, el conocimiento no se recibe pasivamente, ni a través de los sentidos, sino que es construido activamente por el sujeto. Además, la función de la cognición es adaptativa y sirve a la organización del mundo experiencial del sujeto [Von Glassersfeld, 1999], lo que constituye los diferentes aspectos de la subjetividad.

En este nivel se encuentran la mayoría de estudios e investigaciones sobre la cognición musical y el desarrollo de la musicalidad. Estas pesquisas sobre cognición musical también articulan la perspectiva del embodiment, cognición enactiva o corporeizada, según la cual, la experiencia es unificada y delineada por la naturaleza de cada cuerpo y cerebro como un sistema mente-cuerpo que potencia las interacciones del sujeto con su ambiente.

Otro aspecto interesante en este enfoque es el relacionado con los esquemas-imagen corporales que son el soporte de las estructuras sensorio-motrices básicas, por las cuales se halla un mundo comprensible en el que los seres humanos pueden actuar, cuestión que evidencia un patrón dinámico recurrente de las "acciones que se ejercen recíprocamente" [Real Academia Española, 2017] entre el organismo y el ambiente. Pues bien, la cognición enactiva está constituida por interacciones y relaciones sociales, prácticas y artefactos culturales [Maturana, 1997; Johnson, 2007, López-Cano, 2007). Asimismo, la cognición corporeizada aborda el papel de las affordances o diversas posibilidades para la interacción que permite la experiencia musical. Desde la perspectiva de la construcción de las vivencias del individuo y del papel de la música en la generación de paz, este concepto esclarece el estudio de las correlaciones musicales que brindan ciertos contextos; por lo tanto, constituye un elemento interesante para alcanzar el propósito del presente artículo.

El nivel ontogenético de la experiencia musical requiere el desarrollo de una investigación constante y sólida que permita evidenciar los efectos de la música en la construcción de paz, pues esta escasea y, como consecuencia, el discurso referido a la materia se convierte en una especie de propaganda política para justificar la continuación de ciertas prácticas musicales orquestales, y de todo tipo, que no se fundamentan en una reflexión seria sobre el tema.

Por último, aparece el nive/ sociocultura/ que se establece a partir de las interacciones sociales, las cuales originan la función simbólica que está en el centro de la configuración de la experiencia humana. Retomando a Merleau Ponty [2012], la función simbólica se realiza en un proceso de bucle constituido por diversos momentos de percepciónsensación, selección- interpretación-construcción, reflexión-acción, en los cuales ella se constituye como el principal elemento de socialización y, por ende, del principio de construcción de realidad articulada. En palabras de Gilbert Durand [1987], el símbolo es mediador y al mismo tiempo constitutivo de la personalidad por el proceso de individuación. Cassirer, en el texto Filosofía de las formas simbólicas [1976], planteó que la función simbólica se compone de tres elementos: representación, expresión y significación, los cuales se manifiestan en la acción y configuran los diversos sistemas simbólicos que se expresan en forma de lenguajes artísticos. De esta forma, las prácticas artísticas cumplen con las funciones de representar, expresar o significar. Por ello resultan fundamentales en la construcción de paz, pues permiten dar a conocer de diversas maneras una experiencia determinada en varios contextos. 


\section{Experiencia artística y experiencia musical vital}

El estudio de la experiencia musical se fundamenta en la articulación de múltiples perspectivas teóricas [como las de las ciencias cognitivas, la fenomenología, el constructivismo, entre otras] que aportan a la comprensión de la generación de la experiencia humana desde el individuo y sus diferentes niveles [filogenético, ontogenético y sociocultural]. Esto supone basarse en las acciones corporeizadas desde las cuales la escucha, la interpretación y la creación musical constituyen las diversas formas de la experiencia musical.

La perspectiva propuesta por Merleau Ponty [2012] resulta de interés en esta aproximación, porque plantea que la fenomenología describe las estructuras básicas de la experiencia humana, ligándola directamente con la percepción y la sensación [sentidos perceptuales], y con estas, a los recuerdos, asociaciones y anticipaciones que viven los sujetos, lo que la hace extremadamente rica en la construcción de horizontes de significado. Este filósofo articula percepción y experiencia, entendiendo la primera como el fundamento de la subjetividad y la objetividad de la segunda, además, esta última es tanto pasiva como activa, es situacional y práctica, condicionada y libre. Al respecto, señala: "el mundo no es lo que yo pienso, sino lo que yo vivo" [Merleau Ponty, 2012: 20].

En el mismo sentido, pero desde la filosofía de la educación musical, Dewey manifiesta que la experiencia humana es "vitalidad elevada", pues implica un intercambio activo y atento frente al mundo, "significa una completa interpenetración del yo, y el mundo de los objetos y acontecimientos" [2008: 21]. Por lo tanto, para este autor "la naturaleza de la experiencia está determinada por las condiciones esenciales de la vida" [14]. En consecuencia, la construcción del sujeto se va dando en la medida en que este interactúa con su ambiente, lo cual va determinando las vivencias que hacen parte de la historia de su vida. De este modo, la experiencia se configura como un elemento vital. De igual forma, a través de las prácticas artísticas que dan pie a la experiencia misma con el arte, se in-corporan dispositivos [sensaciones, nociones, emociones, etc.] que se hacen posibles por las distintas interacciones, como el contacto con el material artístico, el desarrollo de la creatividad en el juego con un determinado tipo de lenguaje, la posibilidad de expresar y de emocionar, entre otros. Todos factores los cuales permiten in-corporarla o, como diría López [2007], configurar una experiencia enactiva para transformar su componente artístico en experiencia vital.

Otro asunto que se relaciona con la creación de la experiencia artística es el atinente al papel de la emoción, que según Dewey [2008] brinda movilidad y unidad a las partes variadas de una experiencia, convirtiéndola en un todo unificado [aspecto fundamental si se considera el lugar central que ocupa en relación con la construcción de paz].

Desde otra perspectiva, Jean Paul Lederach [2016] en su libro La imaginación moral, advierte que la construcción de paz requiere de imaginación y que esta encuentra su esencia en el mismo acto creativo. Por lo tanto, se requiere una actitud creativa que rompa los moldes para generar paz, explorar nuevos territorios y pensar lo imposible. Los aportes de Lederach [Elliot, 1995] a la articulación entre arte y construcción de paz son de gran valor, sobre todo teniendo presente que la imaginación creativa nos abre nuevos horizontes en relación con los diferentes ambientes posibles para el desarrollo de futuras investigaciones. Este autor propone una analogía entre el acto creativo, la experiencia y la construcción de paz, planteando que:

Arte es aquello que la mano humana toca, moldea y crea, y que, a su vez, toca nuestro más profundo sentido del ser, nuestra experiencia. El proceso artístico posee esa naturaleza dialéctica: surge de la experiencia humana y después da forma, expresión y significado a esa experiencia. La construcción de paz tiene esa misma cualidad artística. [Lederach, 2016: 84]

Este planteamiento evidencia, ya no desde la filosofía, como se expuso anteriormente, sino desde los estudios de paz, la relación entre experiencia artística y construcción de paz: la primera se constituye en espacio dialéctico para la resignificación del sujeto y de las comunidades afectadas por diversas formas de violencia, en camino de aceptar quiénes fueron, quiénes son y quiénes serán. De esta forma, en la propuesta de Lederach [2016] sobre una imaginación moral que se eleva por encima de la violencia [señalando una perspectiva ética], se ubica el lugar de lo individual y lo colectivo (el microsistema y el mesosistema), al observar que, tanto en la experiencia artística como en la construcción de paz, se trata de asumir la responsabilidad personal como también el reconocimiento del carácter recíproco de las relaciones que esto implica.

Aunque es David Elliot [1995) quien desde la filosofía de la educación musical señala los elementos fundamentales para llegar a una conceptualización pertinente de la experiencia musical, explicando que esta depende del entendimiento cultural y contextualmente determinado, tanto en forma tácita como verbal , "de hecho las formas de pensamiento y conocimiento dependientes del contexto median todas las interacciones humanas con los eventos 
físicos que llamamos trabajos musicales" [Elliot, 1995: 125]. De modo que, las experiencias musicales incluyen una variedad de consideraciones porque la musicalidad y los trabajos musicales son construcciones sociales multidimensionales. Por lo tanto, estas siempre incluyen en mayor o menor grado, las realidades sociales de cierto tipo de músicos y de escuchas.

No obstante, Elliot hace un aporte clave cuando plantea: "los trabajos musicales pueden expresar muchos tipos de significados incluyendo significados morales, didácticos, icónicos, políticos, religiosos o personales. De hecho[,] el trabajo de muchas prácticas musicales está usualmente ligado a asuntos de comunicación, entretenimiento, ritual y validación personal y comunitaria" [1995: 125]. Y para explicar mejor el asunto del significado musical, cita a Wayne Bowman [1998], quien afirma que "los significados musicales son negociados, no absorbidos; construidos, no dados; apropiados, no otorgados. Entonces los procesos y experiencias que llamamos musicales nunca residen en un reino estético herméticamente insular, sino que son parte de nuestra realidad social vivida" (1995: 125), y luego añade que "la conciencia sónica no es estática sino temporal y por lo tanto llena de ambigüedad" [Bowman, citado por Elliot, 1995: 127]. Este planteamiento liga directamente la experiencia musical con la producción de sujeto y la significación musical, asuntos relevantes cuando se trata de los pormenores que envuelven los procesos de construcción de paz.

Elliot [1995], también indica que la experiencia musical posee las siguientes características: 1] surge de la relación entre musicalidad y un tipo específico de reto musical; २] los valores fundamentales de ella son el auto crecimiento, autoconocimiento y el disfrute; 3] durante las experiencias musicales, músicos y escuchas usualmente experimentan una concentración focal y absorción profunda; 4] durante el proceso no se necesitan otras motivaciones para mantener la atención y el esfuerzo, aparte de las experiencias de disfrute e integración que surgen de las acciones musicales dirigidas a una meta. Adicionalmente, menciona que las experiencias musicales son lo que Csikszentmihalyi [1998], llama flow experiences o experiencias óptimas, en las que la "presencia continua y fluida del mundo audible penetra la consciencia a un punto que las personas usualmente se sienten positiva o negativamente envueltas, inspiradas o invadidas por una energía sónica" [citado por Elliot, 1995: 126]. El fluir es uno de los componentes importantes de la experiencia musical, en particular cuando esta se transforma en experiencia musical vital, pues la percepción de fluidez se articula con el disfrute y la alegría, lo cual hace que sea una experiencia más vívida, más profunda, en síntesis, una experiencia vital.
De todo lo anterior se desprende que la experiencia musical vital [Zapata y otros, 2004], como vivencia artística posee varias características:1] es experiencia enactiva o, en otras palabras, es corporeizada y, por lo tanto, es experiencia vital, sobre todo en el papel que desempeña en la construcción de paz; 2) surge de la interacción del sujeto con el mundo circundante articulando percepción, sensación y emoción ; 3] posee los valores fundamentales de auto-crecimiento, autoconocimiento y disfrute; 4] está influida por las emociones que dan el sentido de unicidad a la experiencia humana; 4] puede tener un carácter individual o colectivo, pero se sustenta en los diversos tipos de interacciones del individuo, ya sea con otros sujetos, con un material sonoro o con su ambiente; 5] dicha experiencia se constituye en algo que suele afectar y transformar la vida de la persona, le hace posible cambiar de rumbo y mirar la vida con otra perspectiva, esto es, lo que llamamos experiencia musical vital.

En este orden de ideas, la experiencia musical vital reside en el corazón de la experiencia musical y, en relación con la construcción de paz, genera un entramado que puede analizarse en cualquiera de los ámbitos que se exponen a continuación. Su estudio a nivel individual se desarrolla principalmente en el microsistema; a nivel de las interacciones más cercanas para el sujeto, en el mesosistema; a nivel de los conflictos y las políticas locales y regionales, en el macrosistema; y en relación con el exosistema su estudio es posible en el análisis de la influencia de las políticas mundiales en la construcción de paz y en la generación de violencia.

\section{Ámbitos para la investigación sobre la experiencia musical y la construcción de paz}

Con base en lo descrito en los párrafos anteriores, es necesario referir los ámbitos desde los cuales es posible estudiar el rol de las artes y, más específicamente, el de la experiencia musical en la construcción de paz. Es importante señalar que debido a la poca reflexión y a la escasa investigación que hay en este campo, no hay claridad respecto a las diversas prácticas musicales, sus alcances y límites en esta tarea. Gran parte de los discursos sobre construcción de paz en Colombia, se han quedado en la retórica política, instaurándose como una narrativa superficial y estereotipada, llena de lugares comunes, con poco conocimiento de las posibilidades de la experiencia artística y la experiencia musical, sin fundamento en la investigación, basada en prácticas aisladas y en la buena fe de los que las realizan y, por ende, carente de bases sólidas y profundas. 
Por lo tanto, no están analizadas las condiciones específicas, no se han proporcionado evidencias concretas y, debido a la poca indagación, hay aspectos de las relaciones entre música y paz que aún se desconocen. Por lo tanto, quedan muchos temas por estudiar en este terreno, como, por ejemplo, los diversos géneros musicales en relación con diversas poblaciones o las posibilidades que brindan diversas prácticas, géneros o espacios musicales en contextos vulnerables; la importancia de la puesta en escena y los diversos tipos de puesta en escena y el potencial de las interacciones sociales que permite la experiencia musical en personas afectadas por la violencia. Adicionalmente, estos discursos mezclan constantemente los diversos niveles [filogenético, ontogenético y sociocultural] y ámbitos, y, por ende, las repercusiones colectivas e individuales de la violencia en la construcción de sujeto, sin establecer los límites entre unas y otras. Por todo lo anterior argumentan de manera muy general que la música ayuda a los niños de comunidades vulnerables a ser más sociables, pero no explican, con quién, cómo, dónde y tampoco describen el tipo de experiencia musical que ellos tuvieron o los alcances y limitaciones de la misma.

Como lo señaló Humberto de la Calle en 2012 [entrevista en Arcadia):

Aunque el tema del arte en el conflicto no es enteramente nuevo, todavía es un tanto balbuciente. Está en construcción. No hay una sola metodología probada y muchas de sus aplicaciones son experimentales. Pero la herramienta muestra resultados valiosos. La Agencia Colombiana para la Reintegración ha diseñado una estrategia y durante su aplicación ha registrado experiencias exitosas. Un primer hallazgo es que la eficacia depende del quehacer colectivo. No se trata de formar semilleros de artistas de calidad. Se trata de "hacer" el arte para mitigar relaciones antagónicas y asumir el conflicto desde la transformación de la visión del mundo y las relaciones con las demás. Es un abordaje oblicuo por la línea afectiva. Muchas veces no basta la comunicación cognitiva para deconstruir el entramado intelectual/ emocional que recoge y procesa los datos del conflicto. En tales condiciones, el arte no es el fin, no importa la capacidad artística. El arte como herramienta se separa del acto estético para producir efectos en el tejido social.

Estas afirmaciones dejan en claro que aún se requiere de gran trabajo para identificar los aportes del arte en la construcción de paz, pero es necesario tener más claridad sobre las distintas formas como intervienen las prácticas artísticas, ya que no se trata solo de la cohesión social, sino también de la construcción de sujeto y de los desarrollos individuales en un entramado simbólico que nos hace parte de una sociedad.

De modo que es necesario comenzar por diferenciar los distintos ámbitos de la investigación sobre la experiencia musical en la construcción de paz, pues estos tienen que ver con los diversos escenarios posibles de la construcción de sujeto; para ello es preciso distinguir inicialmente entre ámbitos colectivos e individuales. La teoría de Bronfenbrenner [1987] sobre la ecología del desarrollo humano permite ubicar la experiencia artística [y por ende musical] en relación con cierto tipo de espacios y relaciones en la construcción de sujeto. Así, el autor propone que existen diferentes sistemas que influyen en el desarrollo de un individuo, estos pueden representarse como una serie de círculos concéntricos [figura 1], el cual es preciso para ubicar los ámbitos de la investigación que va describir aspectos del desarrollo humano, aunque justamente se retoma para la investigación la perspectiva sistémica de la que subyacen.

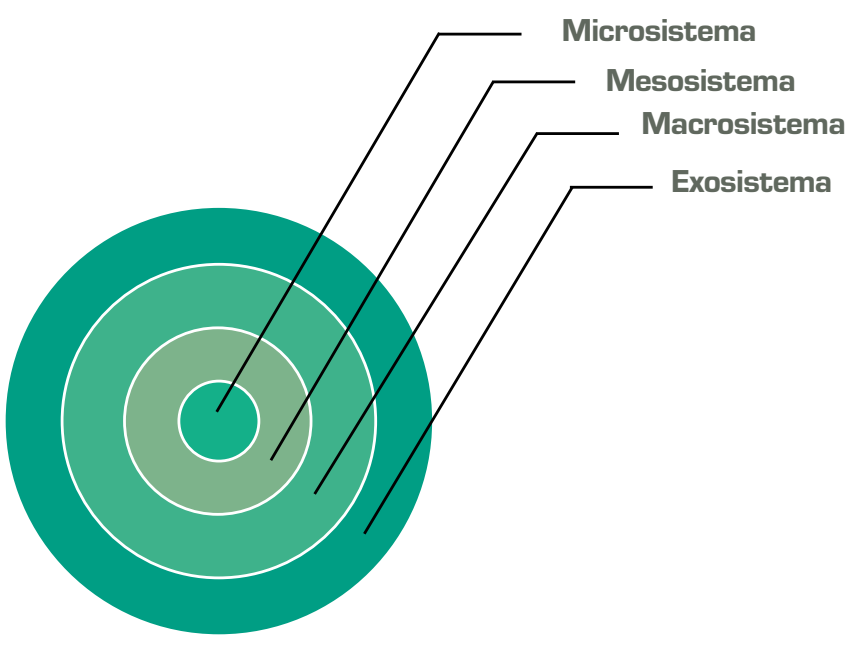

Figura 1. Representación gráfica de la teoría ecológica del desarrollo humano [Brofenbrenner, 1987]

El microsistema corresponde a los espacios de la experiencia individual y el ambiente cercano más íntimo del sujeto, de ahí que el rol de la música se define por los espacios individuales de la experiencia musical tales como: la práctica individual de un instrumento, la escucha musical, la improvisación, la interpretación y la creación musical. En este se desarrolla la mayor parte de los estudios relativos a los efectos psicológicos de la práctica musical. Por otra 
parte, el mesosistema corresponde a las interacciones del individuo en sus espacios vinculares cercanos, como los amigos, la familia o la escuela. En este ámbito se puede plantear el análisis de problemas como el rol de la experiencia musical en el aprendizaje de un instrumento y en las interacciones que permite la práctica colectiva en instituciones sociales, comunitarias y educativas formales y no formales, así como en círculos familiares, el apoyo de la familia o del grupo cercano, y los diversos tipos de intercambios que la experiencia musical permite, entre otros. En relación con la construcción de paz este sistema permite el estudio particular de las formas como influyen la práctica de un instrumento y/o la participación en una orquesta en el desarrollo de conductas como la resiliencia.

El macrosistema constituye el ámbito de lo regionallocal, por ende, el rol de las experiencias musicales en la construcción de paz se ubica en contextos grupales más amplios como, programas orquestales de carácter nacional [ej, "el sistema” o Batuta] o, procesos de negociación entre grupos diversos [como el de las FARC y el gobierno colombiano]. Este nivel también puede abordar las políticas regionales y nacionales que incluyen el arte y la cultura en la construcción de paz.

Finalmente, el exosistema abarca lo internacional y lo mundial. En este sentido, el rol de la experiencia musical en la construcción de paz se ubica en áreas como los derechos humanos, las investigaciones comparadas entre diversos países sobre conflicto y paz, el diseño y aplicación de políticas internacionales sobre arte, cultura y paz, en el trabajo de Organizaciones No Gubernamentales [ONG] y la acción de organismos internacionales como las Naciones Unidas.

Si bien estos ámbitos se conectan entre sí, y en muchos casos se influyen mutuamente para realizar una reflexión profunda que surja del desarrollo de proyectos de investigación, es preciso abordarlos por separado y en relación con los niveles de la experiencia humana a fin de analizar con detalle los alcances o limitaciones de una u otra actividad artística en la transformación creativa del conflicto en distintos contextos [Lederach, 2016], desarrollar líneas y proyectos de investigación constante que permitan hacer una reflexión profunda, combinando acción, práctica y reflexión para superar el discurso estereotipado sobre artes y paz. Aunque en algunos casos también puede articularse un ámbito con otro, esto depende de los objetivos y del tipo de reflexión que se quiera realizar.

También es pertinente mencionar algunos trabajos que desde diferentes enfoques han analizado el rol de la música en la construcción de paz, pues muestran avances en el tema que permiten identificar las tendencias del tema en cuestión. Luján [2016], en un artículo reciente, plantea que es necesario analizar los conflictos y descubrir su complejidad para encontrar soluciones que busquen la mediación, llegando a la conclusión de que la música es un escenario posible para ello, a partir de la revisión de diversos autores nacionales e internacionales que abordan el rol de esta en la construcción de paz y propone que la música ayuda a reconocer diversos agenciamientos que

promueven la cooperación entre grupos diversos en diferentes niveles; musicales, identitarios, económicos, étnicos [interculturales], al encontrar propósitos conjuntos que tienen como finalidad realizar planteamientos respecto a la resolución de diversas tensiones sociales. [Luján, 2016: 3]

El texto de Luján es bastante coherente en la revisión que realiza, sobre todo en relación con el impacto de la música en actividades colectivas que impulsan la cohesión social, la solidaridad intragrupal. También Luján [2016] Ilama la atención sobre el hecho de que la música puede alimentar el conflicto y la violencia; por lo tanto, debe pensarse como herramienta. La reflexión de Luján puede ubicarse en los ámbitos del mesosistema y el macrosistema, aunque, en un pequeño aparte este escrito referencia a Warren [2014] que indica que "la responsabilidad ética de la música surge en el encuentro con el 'Otro' el encuentro cara-a-cara sitúa la percepción que ambos tienen de las relaciones concebidas" [Lujan, 2016: 4), por lo que también sugiere, aunque superficialmente, el ámbito del microsistema y su relación con el mesosistema. Es importante mencionar que Lujan [2016] alude a la corporeidad y la experiencia como aspectos claves en los significados tanto de la música como de la guerra.

En esta misma perspectiva se encuentra el trabajo de Zapata y Hargreaves [2017] en el que se analizan las improvisaciones musicales de niños desplazados entre seis y siete años para observar el efecto de la experiencia musical en su desarrollo socio-afectivo. Los resultados de esta investigación evidencian que las experiencias musicales creativas [juego e improvisación vocal] mejoran el desarrollo socio-afectivo de niños y niñas provenientes de comunidades vulnerables, especialmente en el componente cognitivo de la autoestima.

Otro tipo de investigaciones, como la de Pinto [2014], colocan bajo el punto de vista de las víctimas las letras de las canciones que los victimarios han escrito, y viceversa, 
relacionando el lenguaje verbal y el género musical, lo cual resulta interesante pues brinda diversos aportes a la construcción de memoria histórica y al análisis de la expresión de emociones.

En espacios internacionales, algunas perspectivas como la de Craig Robertson [2010) también indagan por el rol de la música en la construcción de paz. Este investigador realizó una etnografía en Bosnia-Herzegovina con un coro interreligioso [tradiciones católica, ortodoxa y musulmana] y observó que este tipo de actividades corales permiten una tolerancia entre personas de diferentes credos religiosos. Desde la sociología de la música, la propuesta de Robertson se ubica en el ámbito del mesosistema, pues aborda las interacciones y la forma como estas contribuyen al desarrollo de la tolerancia. Con un enfoque similar, pero en el contexto nacional, el trabajo de Rodríguez [2013] tiene como objeto estudiar la cohesión social que la práctica orquestal en Colombia les da a niños y jóvenes pertenecientes al programa Batuta [esta investigación aún se encuentra en etapa de desarrollo].

Todos estos trabajos aportan a la mirada sobre los colectivos y los logros que las personas afectadas por el conflicto pueden alcanzar con diversas experiencias musicales. La mayoría de ellos analizan las interacciones musicales, ubicándose entre los ámbitos del mesosistema y del macrosistema, realmente muy pocos se concentran en los aspectos biológicos, individuales o microsistémicos de la experiencia musical. Por otro lado, casi todos estos escritos han sido realizados en contextos internacionales y solo unos cuantos se han hecho en Colombia, lo cual pone en evidencia la necesidad de indagar mucho más para conocer con mayor claridad los efectos reales de la música en la construcción de paz.

\section{Recursos característicos de los diferentes lenguajes artísticos en la construcción de paz}

La riqueza que brindan las prácticas artísticas en la construcción de paz es variada y crucial. Algunos aspectos relacionados con esta se mencionaron previamente, pero en este aparte se pretende analizar diversas características que son comunes en las prácticas artísticas [y, por ende, también son parte de la experiencia musical vital] y su rol en la construcción de paz. Estos aspectos pueden ser algunos de los ejes sobre los que giren las diferentes acciones e investigaciones que articulan arte y paz.

En relación con los diferentes lenguajes artísticos, se puede señalar el uso de recursos simbólicos y expresivos diferentes, los cuales, como se describió, tienen su fundamento en el trabajo de Cassirer [1976] acerca de los procesos simbólicos y su función representativa, expresiva y simbólica. En este punto es necesario resaltar que gran parte de las experiencias artísticas y, en especial, la experiencia musical vital tiene sentido, pero no tiene un significado explícito, lo que hace que esta posea una ambigüedad en la interpretación, significación y definición exactas [Elliot, 1995; Cross, 2006]. No es posible expresar eso que sucede musicalmente con palabras precisas, por eso el dicho famoso: si pudiera expresarlo con palabras, no lo diría con música. De esta forma, la experiencia musical se constituye en un elemento subjetivo de apropiación y expresión propio del lenguaje sonoro y de los lenguajes no verbales. Este factor permite que todas las expresiones de los sujetos inmersos en una actividad musical [o en una actividad no verbal] sean igualmente válidas e importantes, ya que evidencian la diversidad y riqueza de las formas de comunicación e interacción artística. Adicionalmente, permiten expresar los sentimientos más profundos sin tener que pasar por la definición y precisión que exigen las palabras.

Por ello, la experiencia musical posee un componente hermenéutico que hace posible diferentes interpretaciones del mismo evento artístico. En la construcción de paz estos recursos se constituyen en herramientas cruciales al permitir que los sujetos que interactúan puedan, por ejemplo, expresar todo tipo de emociones [en lugares donde se encuentran grupos en conflicto] sin lugar a ofensas o confrontaciones directas. También pueden representar una emoción profunda sin tener miedo de ser juzgados o recriminados, o redescubrir experiencias dolorosas que estaban guardadas por mucho tiempo.

\section{La metáfora y la metonimia}

Otro recurso propio del lenguaje artístico, que es clave en la construcción de paz, es el uso de la metáfora y la metonimia en la parte expresiva y simbólica. La metáfora y la metonimia permiten usar diferentes imágenes provenientes de la experiencia para expresar, representar o simbolizar una idea, pensamiento, emoción, sentimiento o sensación con el uso del "como si", y con una referencia a otros momentos o percepciones de la misma vivencia. Esto permite recrear en otro escenario lo que explicado directamente con palabras puede ser doloroso o peligroso, y manifestar con todo tipo de imágenes los sentimientos más profundos que están en el interior. Un aspecto importante de la metáfora es que da un sentido poético a la experiencia [Durand, 1987), y por esto, las interacciones se constituyen en escenarios que abren el diálogo y permiten 
la comunicación con cualquiera que comparte la misma vivencia.

\section{Lo performativo}

El aspecto performativo de las experiencias artísticas es crucial, pues transforma una idea en una acción artística, $y$, en muchas ocasiones, permite que las personas salgan de su cotidianidad: fija la atención de los espectadores en aspectos de la vida que parecen habituales y que en los casos de las conductas violentas se han "normalizado". Muchas de los performances se han utilizado para hacer denuncias en lugares donde otras acciones tienen límites. No obstante, aquí se enfatizará en el carácter performativo de las experiencias musicales, ya que implican de hecho una acción que permite que lo simbólico pase al terreno de lo concreto y al in-corporarla se materialice en sonido, movimientos, gestos, imágenes, etc. Esta característica es fundamental en la construcción de paz, pues concede la apropiación y al mismo tiempo la comunicación en diversos espacios, lo cual dinamiza y transforma a los sujetos [activos o pasivos] que en un momento de sus vidas fueron afectados por condiciones de abuso y violencia.

\section{El embodiment o corporeidad}

La experiencia musical implica un in-corporar, representar, un sentir, hacer vida y cuerpo. Eso que se quiere expresar, sin el cuerpo, pierde su carácter subjetivo, personal y de interacción, pues las acciones tenderían a ser solo racionales; se perdería la capacidad de sentir, y en efecto, lo sensible y emocional se tornaría incomprensible y carente de humanidad. La corporeización favorece el hacer propias las experiencias de otros, colocarse en su lugar, y viceversa, y por ende, ayuda a una comprensión más profunda de aquello imposible de expresar con palabras pero que al incorporarlo se comprende en forma tácita.

\section{La puesta en escena}

La mayoría de las acciones artísticas en relación con la construcción de paz incluyen la puesta en escena, los conciertos, las obras de teatro, los performances, y otros géneros que han sido utilizados para la denuncia, la confrontación, el llamado de atención o a la solidaridad con una causa determinada. Entre muchos de los trabajos en esta área, se destaca el de Cohen, Gutiérrez y Walker de la Universidad de Brandeis [2011] en su proyecto Acting Together on the World Stage -Theater Without Borders. Su equipo combina el trabajo de actores y comunidades en la denuncia de abusos y violencia en muchos lugares del mundo. Este proyecto ha tenido impacto mundial y por ello se podría decir que se encuentra en el ámbito del exosistema.

Sin embargo, el tema de la puesta en escena es clave y se debe estudiar a nivel del microsistema, es decir, a nivel de las repercusiones individuales de esta práctica artística. Aunque los efectos de la puesta en escena en el desarrollo de la resiliencia en niños, jóvenes, y en general, en personas afectadas por la violencia, son bastante desconocidos y requieren del desarrollo de una investigación constante. Un elemento esencial de la puesta en escena es que reúne un público, lo cual conlleva el reconocimiento y la visibilización ante otros de una práctica artística. El público se torna en el "otro representativo" para el sujeto en condiciones de vulnerabilidad, y, en muchos casos, brinda una distinción que mejora la autoestima y ayuda en el desarrollo de resiliencia [Zapata y Hargreaves, 2017; Cohen, Gutiérrez y Walker, 2011], ya que se hace visible la situación propia y se comparte con los demás.

Finalmente, la puesta en escena [y en general las prácticas artísticas], posibilitan, entre otras cosas, ubicar en otro lugar la situación que genera el conflicto, sacarla de su ambiente cotidiano y colocarla donde se identifiquen perspectivas diferentes a las que generaron la disputa. En todo caso, se requiere mucha más investigación para conocer sus alcances y limitaciones.

\section{La creación e improvisación}

El aspecto creativo de las prácticas artísticas es uno de los elementos que más relevancia tiene en la construcción de paz, pues promueve el juego, el conocimiento y la comunicación, con otros lenguajes. En una investigación reciente [Zapata y Hargreaves, 2017] se encontró que la improvisación musical es un elemento fundamental en la autoestima de niños de seis años provenientes de comunidades vulnerables, a través de un taller en el que se emplearon juegos e improvisaciones vocales durante ocho meses y en sus resultados se notó que el aspecto más afectado por las actividades musicales fue el componente cognitivo de la autoestima. Al parecer, el manejo y disfrute de la expresión con otro tipo de lenguaje permite a los niños desarrollar confianza y seguridad en sí mismos.

En este orden de ideas, y a manera de corolario, es preciso señalar que los elementos básicos de las prácticas artísticas poseen unas funciones esenciales en la construcción de paz, algunas que se pueden nombrar aquí muy someramente son: 
- $\quad$ Expresión de las situaciones vividas y apertura al diálogo.

- $\quad$ Denuncia y visibilización de problemas, resistencias, abusos y conflictos.

- Invitación a grupos en conflicto al diálogo y a la construcción de acuerdos para reconocer y reconocerse, celebrar y disfrutar en otros espacios.

- $\quad$ Transformación individual y colectiva.

- Recuperación de la confianza y transformación de las interacciones.

- Desarrollo de resiliencia, autoestima, autoconocimiento, autocrecimiento, socialización y disfrute, entre otros.

Finalmente, un elemento importante de la relación arte y construcción de paz, [como se indicó anteriormente], es la humanización de los conflictos. Por ello, un aspecto fundamental en estos procesos es el emocional o socio afectivo. Las investigaciones que han estudiado el rol de las artes en el desarrollo socio afectivo y emocional evidencian que las prácticas artísticas tienen un papel preponderante en la construcción de sujeto y por ende en el desarrollo de la autoestima, el autoconocimiento, el auto crecimiento, el disfrute y los procesos de socialización [Elliot, 1995; Zapata y Hargreaves, 2017].

Retomando a Elliot [1995], uno de los aspectos más relevantes en este campo, es el aporte que las artes hacen al sujeto en el reconocimiento de sí mismo y del otro como ser vivo, sensible y cognoscente, en otras palabras, como ser humano. Las prácticas artísticas, cuando están ligadas a lo vital, están dirigidas a recuperar la humanidad propia y de los demás, llevan a descubrir al otro en una dimensión más integral, como ser humano que siente, que sufre, que se alegra, que disfruta y juega, esto es, como ser que vive, en una frase, generan la oportunidad de ver al otro no como objeto, sino como persona.

Este aspecto es crucial en la construcción de paz, ya que se trata del reconocimiento del otro como ser diferente que puede pensar y sentir, pero que también tiene sentimientos, emociones, dificultades, derechos y una vida como la de cualquiera, es decir, se reconoce al otro como igual. Volviendo a lo propuesto en los párrafos previos, la experiencia musical vital gira alrededor de estas cuestiones del desarrollo socio afectivo: en el reconocimiento de sí mismo y del otro como seres humanos en toda su dimensión. Por lo tanto, es desde el componente psicosocial, que por medio de la música y en conexión con el contexto familiar, educativo y sociocultural, se desarrollan conductas resilientes y de superación de las dificultades ocasionadas por las situaciones de violencia.

Como conclusión, para ubicar el rol de las artes en la construcción de paz hay bastante trabajo por hacer, pues no solo se trata de la labor que desarrollan las ONGs, las organizaciones de activistas y los artistas en sus obras, también quedan retos tan importantes como el desarrollo de investigaciones rigurosas que permitan desde los diferentes ámbitos, identificar qué rol tiene cada uno de los lenguajes artísticos y dentro de cada uno de estos, esclarecer cuáles elementos y recursos simbólicos inciden en qué tipo de poblaciones y en qué clase de traumas causados por las distintas formas de violencia. Así que, hay un largo camino por recorrer en la búsqueda de resultados en esta materia. Como lo señaló Humberto de la Calle en una entrevista realizada por la revista Arcadia:

Pero es en la fase posterior de aclimatación de la paz donde la expresión artística despliega su mayor potencial. Terminado el conflicto, se abre la etapa de la aclimatación de la paz. Es el momento de las transformaciones de la sociedad[...] Terminado el conflicto, la lucha se moverá hacia otras esferas como se dijo, y una de ellas, sin duda, será la lucha por la narrativa. Es más, puede decirse que esa será la batalla final. La narrativa, sin embargo, aunque se emparenta con la verdad, no se construye sólo a base de ella. Tan importante como la verdad, en términos de confrontación, es la leyenda, la exageración, el mito. El heroísmo. [2012]

Esto advierte el compromiso y los retos que están presentes en el campo de las artes para la construcción de paz, y destaca que aún hay un largo camino por seguir en la obtención de una paz sostenible y en la reflexión sobre el rol de las artes en ese proceso.

\section{Referencias}

Bowman, W. [1998]. Philosophical Perspectives on Music. New York: Oxford University Press.

Bronfenbrenner, U. [1987]. La ecología del desarrollo humano. Barcelona: Paidós.

Cassirer, E. [1976]. Filosofía de las formas simbólicas. México: Fondo de Cultura Económica.

Cohen, C., Gutiérrez, R. and Walker, P. [2011]. Acting 
Together I: Performance and Creative Transformation of Conflict. Boston: Newvillage Press.

Cross, I. [2006]. "Music and Social Being”. En: Musicology Australia, [28], p.114-126.

Csikszentmihalyi, M. [1998]. Creatividad. El fluir y la psicología del descubrimiento y la invención. Barcelona: Paidós.

De la Calle, H. [2012], Entrevista en Revista Arcadia, recuperado de http:/ / www.revistaarcadia.com/agenda/ articulo/de-la-calle-habla-por-primera-vez-del-papel-de-lasartes-en-el-proceso-de-paz/35856. Consultado el 15-22017

Dewey, J. [2008]. El arte como experiencia. Barcelona: Paidós.

Durand, G. [1987]. La imaginación simbólica. Buenos Aires: Amorrortu editores.

Elliot, D. [1995]. Music Matters. A new Philosophy of Music Education. New York: Oxford University Press.

Lederach, J. P. [2016]. La imaginación moral. El arte y el alma de la construcción de paz. Bogotá: Semana Libros.

Llinás, R. [2003]. El cerebro y el mito del yo. Bogotá: Editorial Norma.

López, R. [2007]. "Semiótica, semiótica de la música y semiótica cognitivo-enactiva de la música. Notas para un manual de usuario", recuperado de http:/ / rlopezcano. blogspot.com.co/p/publicaciones-ruben-lopez-cano-1996.

Consultado el 13 de diciembre de 2016.

Luján, J. D. [2016]. "Escenarios de no-guerra: el papel de la música en la transformación de sociedades en conflicto".

En: Revista CS, [19], pp. 167-199. Cali, Colombia: Facultad de Derecho y Ciencias Sociales, Universidad Icesi.

Maturana, H. [1976]. El árbol del conocimiento. Santiago de Chile: Editorial Universitaria.

[1997]. De máquinas y seres vivos,

autopoiesis de la organización de lo vivo. Santiago de Chile: Editorial Universitaria.

[2002]. Transformación en la convivencia.

Santiago de Chile: Dolmen Ediciones.

Malloch, S. and Trevarthen C. [eds] [2009]. Communicative
Musicality: Exploring the basis of Human Companionship. Oxford University Press.

Merleau-Ponty, M. [2012] Phenomenology of Perception. New York: Routledge.

Pinto, M. E. [2014]. "Music and Reconciliation in Colombia: Opportunities and Limitations of Songs Composed by Victims". En Music and Arts in Action, 4 [2]. http:/ / www. musicandartsinaction.net/index.php/maia/article/view/ musicreconciliationcolombia/97 Consultado 13-12- 2016.

Robertson, C. [2010]. Music and Conflict Transformation in Bosnia: Constructing and Reconstructing the Normal. Music and Arts in Action, 2 [२], recuperado de http:/ / musicandartsinaction.net/index.php/maia/article/view/ conflicttransformationbosnia.

Real Academia Española [2017]. Interacción, recuperado de http:/ / dle.rae.es/?id=LsCpk2t. Consultado el 20-22017.

Rodríguez, A. [2013]. "Los programas musicales colectivos como espacios de construcción de paz. Caso Programa Música para la Reconciliación de la Fundación Nacional Batuta en Colombia”, recuperado de http:/ / repositori.uji. es/xmlui/handle/10234/132046. Consultado el 13-122016.

Warren, J. R. [2014]. Music and Ethical Responsibility. Cambridge, United Kingdom: Cambridge University Press.

Zapata, G. P., B. Goubert, y J. F. Maldonado [2004].

Universidad, músicas urbanas, pedagogía y cotidianidad. Bogotá: Universidad Pedagógica Nacional.

Zapata, G. P. y D.J. Hargreaves [2017]. "Musical identities, resilience and wellbeing: The effects of music on displaced children in Colombia”. En Handbook of Musical Identities. MacDonald, R. Hargreaves, J. D. \& Miell, D. [2017], chapter 40. Oxford: Oxford University Press. 